\title{
Integrated characterization and validation of the prognostic significance of microRNA-200s in colorectal cancer
}

Qiliang Peng ${ }^{1,2 \dagger}$, Ming Cheng ${ }^{3 \dagger}$, Ting $\mathrm{Li}^{1,2}$, Xiangying Chen ${ }^{1,2}$, Yi Shen ${ }^{4}$, Yaqun Zhu ${ }^{1,2}$ and Bo Xu ${ }^{3^{*}}$ (D)

\begin{abstract}
Background: Accumulating evidence has demonstrated that microRNA-200s (miR-200a, miR-200b and miR-200c) could serve as promising molecular biomarkers for cancer prognosis. Nevertheless, the associations between miR200s expression and colorectal cancer (CRC) prognosis remain controversial.
\end{abstract}

Methods: We applied two mainstream approaches combining meta-analysis and bioinformatics analysis to answer whether miR-200s were associated with the prognosis of CRC patients and why miR-200s could be used as prognostic biomarkers for CRC.

Results: Consequently, low expression of miR-200s was associated with unfavorable overall survival (OS) in CRC patients (HR: 1.09; 95\% Cl 1.01-1.17; P = 0.025). According to the subgroup analysis, the prognostic role of miR-200s was more significant for tissue samples, large samples, American patients and miR-200a subgroups. Then the target genes of miR-200s were predicted and applied for functional enrichment analyses. The results showed that the target genes of miR-200s were mainly enriched into some vital ontology subjects such as regulation ability, key cell structures and binding function. Moreover, a series of important signaling pathways were identified, which were significantly linked with the initiation and progression of CRC. Additionally, a protein-protein interaction (PPI) network of miR-200s targets was constructed to screen hub genes and modules. The identified hub genes and modules were validated to be highly involved in the occurrence and development of CRC.

Conclusions: Current evidences revealed that miR-200s could be promising biomarkers for CRC prognosis. However, the findings still need to be validated with more larger-scale prospective studies and biological experiments before miR-200s could be applied into clinical application.

Keywords: Colorectal cancer, Biomarker, Prognosis, Bioinformatics

\section{Background}

Colorectal cancer (CRC) remains as one of the most malignant cancer types across almost all the countries and regions because of the marked morbidity and

\footnotetext{
${ }^{*}$ Correspondence: sdfeyxubo@163.com

${ }^{\dagger}$ Qiliang Peng and Ming Cheng contributed equally to this work

${ }^{3}$ Department of General Surgery, The Second Affiliated Hospital

of Soochow University, San Xiang Road No. 1055, Suzhou, Jiangsu 215004, China

Full list of author information is available at the end of the article
}

mortality [1]. Despite the advancement of novel treatment options and early detection, the survival outcome of CRC patients has not improved significantly [2]. Current cancer surveillance and management mainly depend on the tumor makers such as CEA and imaging information such as computed tomography (CT) based on tumor node metastasis (TNM) stage and grade [3]. However, more effective prognostic markers and therapeutic methods applied for CRC treatment and prevention have still not been identified, which prevents the precise prediction of the prognosis and metastasis of CRC patients.

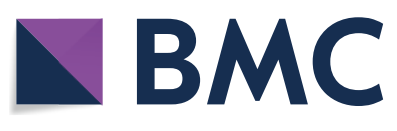

(c) The Author(s) 2020. This article is licensed under a Creative Commons Attribution 4.0 International License, which permits use, sharing, adaptation, distribution and reproduction in any medium or format, as long as you give appropriate credit to the original author(s) and the source, provide a link to the Creative Commons licence, and indicate if changes were made. The images or other third party material in this article are included in the article's Creative Commons licence, unless indicated otherwise in a credit line to the material. If material is not included in the article's Creative Commons licence and your intended use is not permitted by statutory regulation or exceeds the permitted use, you will need to obtain permission directly from the copyright holder. To view a copy of this licence, visit http://creativeco mmons.org/licenses/by/4.0/. The Creative Commons Public Domain Dedication waiver (http://creativecommons.org/publicdomain/ zero/1.0/) applies to the data made available in this article, unless otherwise stated in a credit line to the data. 
Thus, there is an urgent need for discovering valuable molecular biomarkers with high precision and noninvasive nature for promoting early screening, prognostic classification, and promising therapeutic strategies for CRC [4].

The discovery of microRNAs (miRNAs), which have been identified as prominent and intriguing tumor biomarkers for noninvasive tests, has provided new ideas for early cancer diagnosis and survival prediction [5]. In general, miRNAs are a class of evolutionarily conserved, endogenous small noncoding with length of 18-24 nucleotides that could influence a variety of critical cellular processes, including cell growth, differentiation, proliferation, apoptosis and metabolism [6]. There is increasing published evidence that deregulated miRNAs with aberrant expression levels were highly involved in cancer initiation and progression and could be a novel kind of biomarkers for various cancer types including CRC [7].

Interestingly, the miR-200s (miR-200a, miR-200b and miR-200c) are typical and most extensively studied examples in functional miRNAs. Recent studies have indicated that miR-200s were dysregulated in various human cancers including breast cancer, ovarian cancer, prostate cancer, esophageal cancer, gastric cancer and CRC [8]. In $\mathrm{CRC}$, there have been several studies validating the association between an aberrant expression level of miR-200s and the survival outcome [9]. Nevertheless, there were still studies revealing the insignificant or opposite results. Moreover, the understanding of the underlying mechanism of miR-200s involved in abnormal development of CRC is still not fully elucidated while this is very important to explain the diagnosis, treatment, prognosis and prevention of this disease.

Therefore, it is essentially necessary to conduct this systematic and comprehensive literature search of all eligible studies for summarizing the published global findings, and obtaining overall prognostic significance of miR-200s expression in CRC. Additionally, we also performed an integrated bioinformatics analysis to uncover the potential mechanisms of miR-200s involved in CRC and to get a better understanding on the biomarker roles of miR-200s.

\section{Materials and methods}

\section{Literature search strategy}

The part of meta-analysis was carried out totally based on the guidelines of Preferred Reporting Items for Systematic Reviews and Meta-Analyses (PRISMA) [10]. To accomplish a comprehensive literature search, we applied several databases including PubMed, EMBASE, Web of science, and the Cochrane Library with the following combination keywords: "microRNA-200a or microRNA$200 \mathrm{~b}$ or microRNA-200c or miR-200a or miR-200b or
miR-200c or miRNA-200a or miRNA-200b or miRNA$200 \mathrm{c}$ " and "cancer or tumor or carcinoma or neoplasm" or "colorectal or colon or rectal or rectum". We conducted the computerized search up to October 26th, 2019. Additionally, the reference lists of the relevant articles were independently scanned for the potentially additional studies.

\section{Inclusion and exclusion criteria}

Studies complied with the following criteria were considered eligible: (1) the study explored prognostic value of miR-200s expression in CRC patients; (2) histologic assessment was used as gold standard for CRC; (3) the study provide prognostic indices such as hazard ratios (HRs) and the corresponding 95\% confidence intervals (CIs), or sufficient information to calculate these parameters. Exclusion criteria for the articles included: (1) not related to our research topic; (2) without valuable data for our final data synthesis; (3) the forms of letters, reviews, case reports, conference papers or editorials.

\section{Data extraction and document assessment}

Two researchers independently extracted key information with standardized forms, and discrepancies were settled by a third investigator. The following data and information were collected from each study: first author, publication year, sample source, detection method, patient population, patient number, tumor stage, followup time, outcome measures, HR, and corresponding 95\% CI. If the HRs and 95\% CIs were not directly determined by univariate and multivariate analyses, they may be extracted from provided Kaplan-Meier curves according to methods developed by Tierney et al. [11]. Methodological qualities of selected studies were evaluated by the Newcastle-Ottawa Scale (NOS) method [12]. The NOS scores ranged from 0 to 9 ; by convention, a study was considered as high quality if it has the NOS score $>6$.

\section{Statistical methods for data synthesis}

All of the HRs and corresponding 95\% CIs from included studies were applied to estimate the pooled HR for obtaining a whole standpoint of the associations between miR-200s expression and survival outcome. Inter-study heterogeneity was judged by using the Cochran $Q$ and $\mathrm{I}^{2}$ test [13]. According to the practice, a P-value of less than 0.05 for the $Q$ test or an $\mathrm{I}^{2}$ value of more than $50 \%$ was regarded as indicators of high heterogeneity. The random-effects model would be estimated for the studies with a significant heterogeneity $\left(\mathrm{P}_{\mathrm{Q}} \leq 0.05\right.$; $\mathrm{I}^{2} \geq 50 \%$ ); Otherwise, a fixed effects model would be applied $\left(\mathrm{P}_{\mathrm{Q}}>0.05 ; \mathrm{I}^{2}<50 \%\right)$. Then the subgroup analysis, the meta-regression analysis and the sensitivity analysis were conducted to explore the potential sources of 
heterogeneity [14]. Finally, Begg's funnel plot and Egger's tests were applied to assess the included studies for the possibility of publication bias [15]. All statistical tests in the meta-analysis part were carried out using STATA 12.0 software. A value of $\mathrm{P}<0.05$ was suggested statistically significant.

\section{Identification of target genes}

The target genes of miR-200a, miR-200b and miR-200c were respectively predicted from the powerful database: miRTarBase, which was designed for comprehensive information regarding experimentally proved miRNAtarget interactions [16]. In the most recent update, the database has been recognized as one of the most integrative annotated and experimentally proved miRNA-target interaction tools with more than 13,404 proved miRNAtarget interactions from 11,021 articles [17].

\section{Gene ontology and pathway enrichment analysis}

To understand why miR-200s were associated with occurrence and development with CRC, functional enrichment analyses of the targets of miR-200s were performed including Gene Ontology (GO) analysis and Kyoto Encyclopedia of Genes and Genomes (KEGG) pathway analysis $[18,19]$. Gene ontology analyses concentrate on three domains including biological processes, cellular components, and molecular functions. To accomplish GO and KEGG analysis of the target genes of miR-200s, the online tool Database for Annotation, Visualization and Integrated Discovery (DAVID) was applied [20]. The DAVID online software identified the significant function terms and potential pathways of the target genes with $\mathrm{P}$-values $<0.05$.

\section{Construction and analysis of the PPI network}

The protein-protein interaction (PPI) information were retrieved from the Search Tool for the Retrieval of Interacting Genes (STRING) database, which is an online tool developed for collecting, scoring and integrating all publicly available sources of PPI data, and complementing these with computational predictions [21]. The target genes of miR-200s were mapped to STRING to evaluate the PPI information and data with confidence scores $>0.4$ were regarded as significant and retrieved. Then Cytoscape was employed to analyze and visualize the network. The hub genes were selected using the CytoNCA network analyzer plug-in based on three different centrality measures including betweenness centrality and closeness centrality and degree centrality [22]. Moreover, the MCODE plug-in was utilized to filter and identify the significant network modules in Cytoscape. Finally, KEGG functional analysis was performed to further determine the function of these hub genes and genes involved the identified modules. $\mathrm{P}<0.05$ was regarded to indicate a statistically significant result.

\section{Results}

Summary of the included studies

The literature identification process was depicted in detail at Fig. 1. A total of 381 records were identified through systematic search and manual review for initial search. On the basis of the study selection and exclusion criteria, finally, a total of 11 articles including 30 studies were found to be eligible and enrolled in the data synthesis [23-33]. Overall, all enrolled studies obtained a NOS score more than 6 , which revealed a moderately satisfactory quality. The studies were published from 2006 to 2019. The participants of the selected articles were Asians, Europeans and Americans. Most patients had a relatively long follow-up time. The specimens used in these 30 studies were classified as blood $(n=9)$, and tissue $(n=21)$. The expression levels of miR-200s were evaluated via quantitative reverse transcription polymerase chain reaction (RT-PCR). The main characteristics of the 16 studies eligible for meta-analysis are shown in Table 1.

\section{Identification of the prognostic value of miR-200s}

As obvious heterogeneity was identified among the enrolled researches $\left(\mathrm{I}^{2}=70.6 \%, \mathrm{P}<0.001\right)$, subsequent meta-analysis with a random-effect model was applied to generate the pooled OS. The results demonstrated that low expression of miR-200s was significantly linked with poor OS (HR: 1.09; 95\% CI 1.01-1.17; P=0.025; Fig. 2), compared than those patients with high expression of miR-200s.

Given that the substantial heterogeneity exhibited, subgroup analysis was performed to explore the heterogeneity of covariates including sample source, sample size, ethnicity and miR-200s classification (Table 2). Sample source subgrouping explored that low miR-200s expression predicted significant poor OS in tissue samples (HR: 1.16 ; 95\% CI 1.08-1.26; $\mathrm{P}<0.001$ ), but not in blood samples (HR: 0.89; 95\% CI 0.76-1.05; $\mathrm{P}=0.161$ ). Subgroups analysis by ethnicity indicated that lower miR-200s expression status was identified as a significant worse prognostic marker in Americans (HR: 1.19; 95\% CI 1.08-1.30; $\mathrm{P}<0.001$ ), but not in Asians (HR: 0.69; 95\% CI 0.23-2.01; $\mathrm{P}=0.491$ ) and Europeans (HR: 0.98 ; $95 \%$ CI $0.87-1.10 ; \mathrm{P}=0.707)$. Subgroups analyses were also carried out based on sample size and the results revealed that the predictive effect was more significant in large sample size $(H R=1.06$; $95 \%$ CI $1.01-$ 1.12; $\mathrm{P}=0.036)$ than in small sample size $(\mathrm{HR}=1.17$; 95\% CI $0.94-1.46 ; \mathrm{P}=0.159$ ). When stratified by miR200s classification, the prognostic role of miR-200s was more significant for miR-200a with the combined HR 


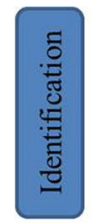

\begin{tabular}{l}
$\begin{array}{l}\text { Records identified through } \\
\text { database searching } \\
(\mathrm{N}=368)\end{array}$ \\
\hline
\end{tabular}

Additional records identified

through other sources

$(\mathrm{N}=368)$

$(\mathrm{N}=13)$

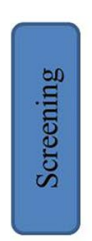

Records reviewed for duplicates

$(\mathrm{N}=381)$

137 duplicates among databases

Records after removing duplicates

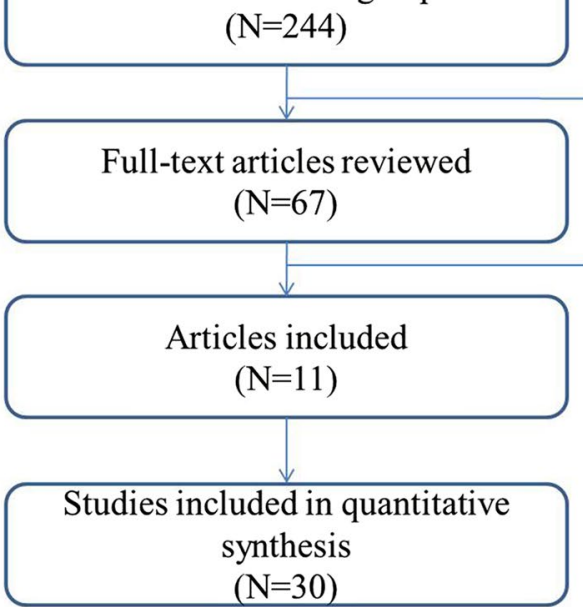

177 articles were excluded:

Letters, reviews, meta-analyses

Not about human studies

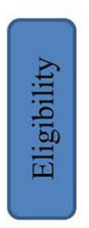

Not related to our topic

56 articles were excluded:

Not relevant to prognosis

Without sufficient data

With duplicate data

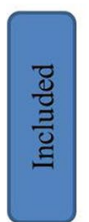

$(\mathrm{N}=30)$

Fig. 1 A flowchart presenting the steps of literature retrieval and selection

being 1.13 (95\% CI 1.01-1.26; $\mathrm{P}=0.027)$ than $\mathrm{miR}-200 \mathrm{~b}$ $(\mathrm{HR}=1.14 ; 95 \%$ CI $0.97-1.33 ; \mathrm{P}=0.113)$ and $\mathrm{miR}-200 \mathrm{c}$ $(\mathrm{HR}=1.00 ; 95 \% \mathrm{CI} 0.85-1.17 ; \mathrm{P}=0.973)$.

Meta-regression analysis was further carried out based on the main characteristics. The meta-regression revealed that heterogeneity may not be induced by sample source $(P=0.196)$, sample size $(P=0.124)$, ethnicity $(\mathrm{P}=0.136)$ and $\mathrm{miR}-200$ s classification $(\mathrm{P}=0.656)$, as the results have not reached statistical significance.

\section{Sensitivity analysis and publication bias}

To investigate the robustness of relationship and explore potential factors of statistical heterogeneity, sensitivity analyses were carried out by skipping at a time to evaluate the specific influence of the individual data on the pooled HRs. Sensitivity analysis demonstrated that no obvious variation in pooled HR by excluding any of the study, suggesting that the conclusions are stable (Fig. 3). Begg's funnel plot and Egger's tests were employed to evaluate the publication bias. The value was 0.40 for Egger's tests, which revealed there was no obvious evidence of publication bias (Fig. 4).

\section{Functional characterization of miR-200s}

To understand the miR-200s underlying biological processes and pathways, GO and KEGG pathway analyses were performed to explore the biological functions. The top 10 enriched results of GO analysis were presented at Fig. 5. With respect to biological processes, the target genes of miR-200a, 200b and miR-200c were all primarily enriched in regulating some important biological processes such as regulation of transcription from RNA polymerase II promoter, regulation of cell proliferation and regulation of apoptotic process. Regarding the enriched cellular components, the target genes of miR200a, 200b and miR-200c were all mainly clustered in some key cell structures including nucleus, cytoplasm and nucleoplasm. In addition, with regard to molecular function, the target genes of miR-200a, 200b and miR200c were all highly involved in binding function containing transcription regulatory region DNA binding, protein binding, and transcription factor binding.

The top significantly enriched KEGG pathways for the target genes of miR-200a, miR-200b and miR-200c were also identified and plotted at Fig. 6. These genes may play a crucial role in several important pathways including miRNAs in cancer, pathways in cancer, FoxO signaling 


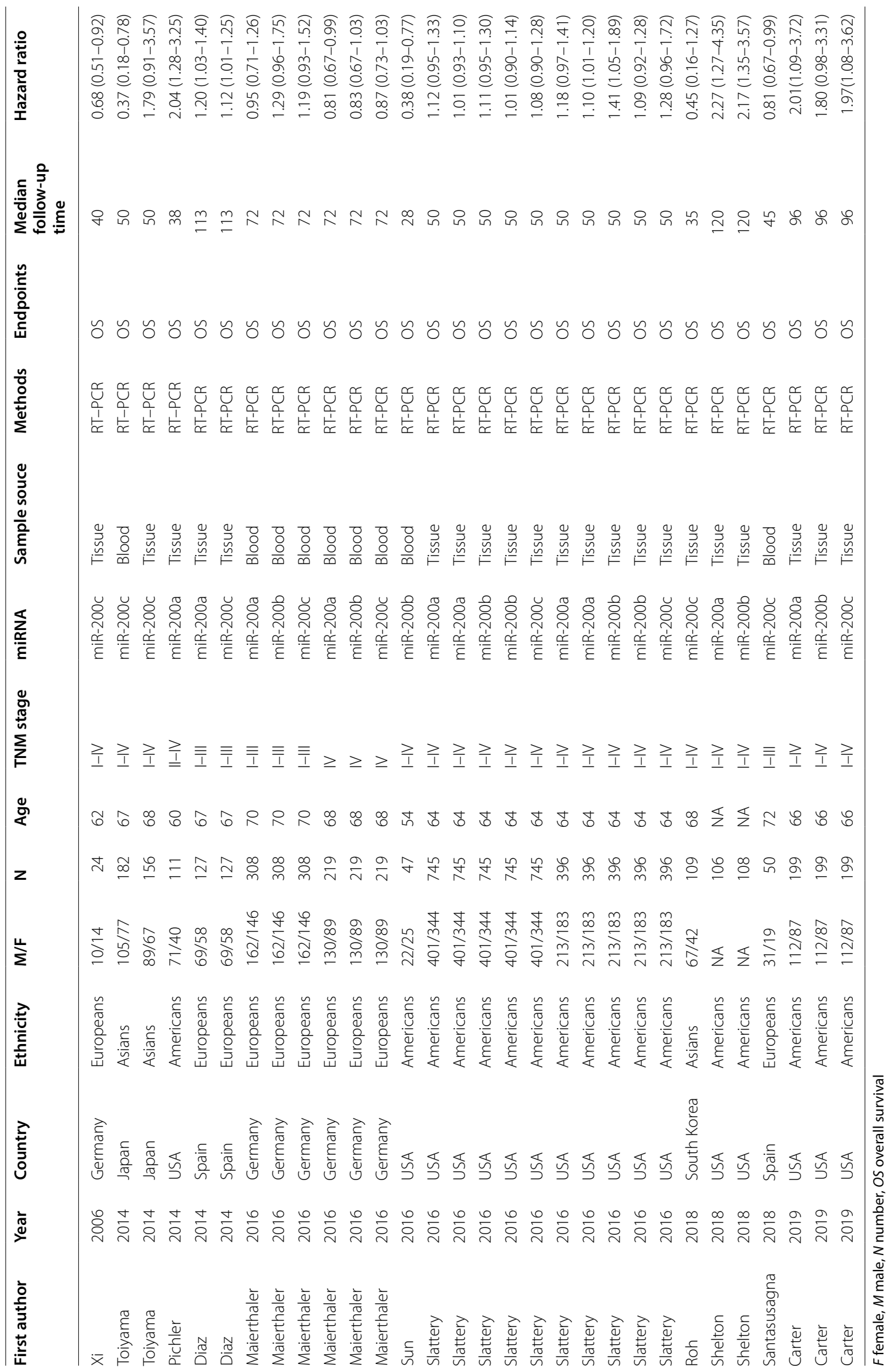




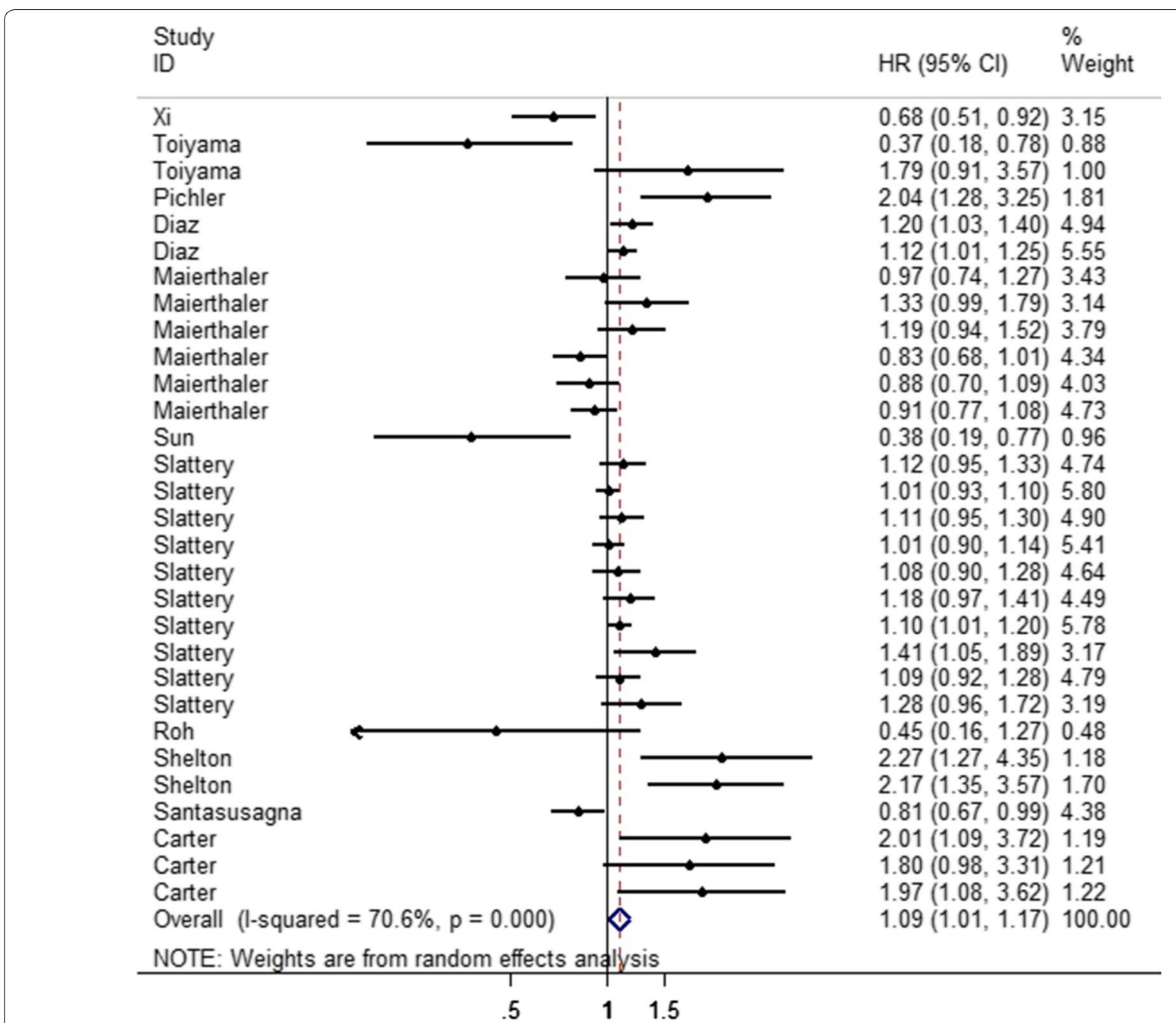

Fig. 2 Forest plot of studies evaluating the correlation of low miR-200s expression with overall survival of colorectal cancer patients

pathway, proteoglycans in cancer, colorectal cancer, p53 signaling pathway, PI3K-Akt signaling pathway, HIF-1 signaling pathway and focal adhesion. As shown in Fig. 7, FoxO signaling pathway, one of the most representative pathways, also had close relations with cell cycle, apoptosis, regulation of autophagy, MAPK signaling, PI3K-Akt signaling and JAK-STAT signaling pathway.

\section{Integration of protein-protein interaction network}

To further investigate the potential associations among the target genes of miR-200s, we constructed the PPI networks of the targets of miR-200a, miR-200b and miR$200 \mathrm{c}$, respectively. By separately uploading the targets of miR-200a, miR-200b and miR-200c into STRING database, the PPI networks of miR-200a, miR-200b and miR200c were visualized using Cytoscape. To identify the most important target genes of miR-200s, the Cytoscape CytoNCA Network Analyzer plug-in identified top ten hub genes from the PPI networks of miR-200s by estimating the network nodes based on centrality analysis (Fig. 8). In detail, TP53, EGFR, PTEN, CTNNB1, STAT3, HSPA4, SMAD2, EZH2, HGF, SOD2 were identified for miR-200a, KRAS, VEGFA, EP300, JUN, ZEB1, FN1, RHOA, EZH2, NOTCH1, KDR were screened for miR200b, and EP300, PTEN, VEGFA, FN1, CDH1, NOTCH1, KRAS, JUN, MDM2, HSP9OAA1 were retrieved for miR200c. Comprehensive function analysis indicated that the hub genes for miR-200a were significantly associated with FoxO signaling pathway, pathways in cancer, proteoglycans in cancer, miRNAs in cancer, colorectal cancer, and PI3K-Akt signaling pathway, the hub genes for miR-200b were mainly involved in Focal adhesion, Notch signaling pathway, VEGF signaling pathway, and Wnt signaling pathway while the hub genes for miR-200c were highly linked with pathways in cancer, miRNAs in cancer, PI3K-Akt signaling pathway, FoxO signaling 
Table 2 Results of subgroup and meta-regression analyses

\begin{tabular}{|c|c|c|c|c|c|c|}
\hline Subgroup & Studies & $\mathrm{HR}(95 \% \mathrm{Cl})$ & P-value & Heterogeneity $\left(\mathrm{I}^{2}\right)(\%)$ & $P_{\text {heterogeneity }}$ & $\begin{array}{l}\text { Meta- } \\
\text { regression } \\
\text { (P-value) }\end{array}$ \\
\hline Sample source & & & & & & $P=0.196$ \\
\hline Blood & 9 & $0.89(0.76-1.05)$ & $P=0.161$ & 68.5 & $P=0.001$ & \\
\hline Tissue & 21 & $1.16(1.08-1.26)$ & $P<0.001$ & 64.4 & $P<0.001$ & \\
\hline Sample size & & & & & & $P=0.124$ \\
\hline Large (> median) & 16 & $1.06(1.01-1.12)$ & $P=0.036$ & 40.5 & $P=0.047$ & \\
\hline Small (<median) & 14 & $1.17(0.94-1.46)$ & $P=0.159$ & 82.1 & $P<0.001$ & \\
\hline Ethnicity & & & & & & $P=0.136$ \\
\hline Asian & 3 & $0.69(0.23-2.01)$ & $P=0.491$ & 81.3 & $P=0.005$ & \\
\hline Europeans & 10 & $0.98(0.87-1.10)$ & $P=0.707$ & 72.1 & $P<0.001$ & \\
\hline Americans & 17 & $1.19(1.08-1.30)$ & $P<0.001$ & 67.1 & $P<0.001$ & \\
\hline miR-200s classification & & & & & & $P=0.656$ \\
\hline miR-200a & 10 & $1.13(1.01-1.26)$ & $P=0.027$ & 69.6 & $P=0.001$ & \\
\hline miR-200b & 9 & $1.14(0.97-1.33)$ & $P=0.113$ & 72.3 & $P<0.001$ & \\
\hline miR-200c & 11 & $1.00(0.85-1.17)$ & $P=0.973$ & 74.0 & $P<0.001$ & \\
\hline
\end{tabular}

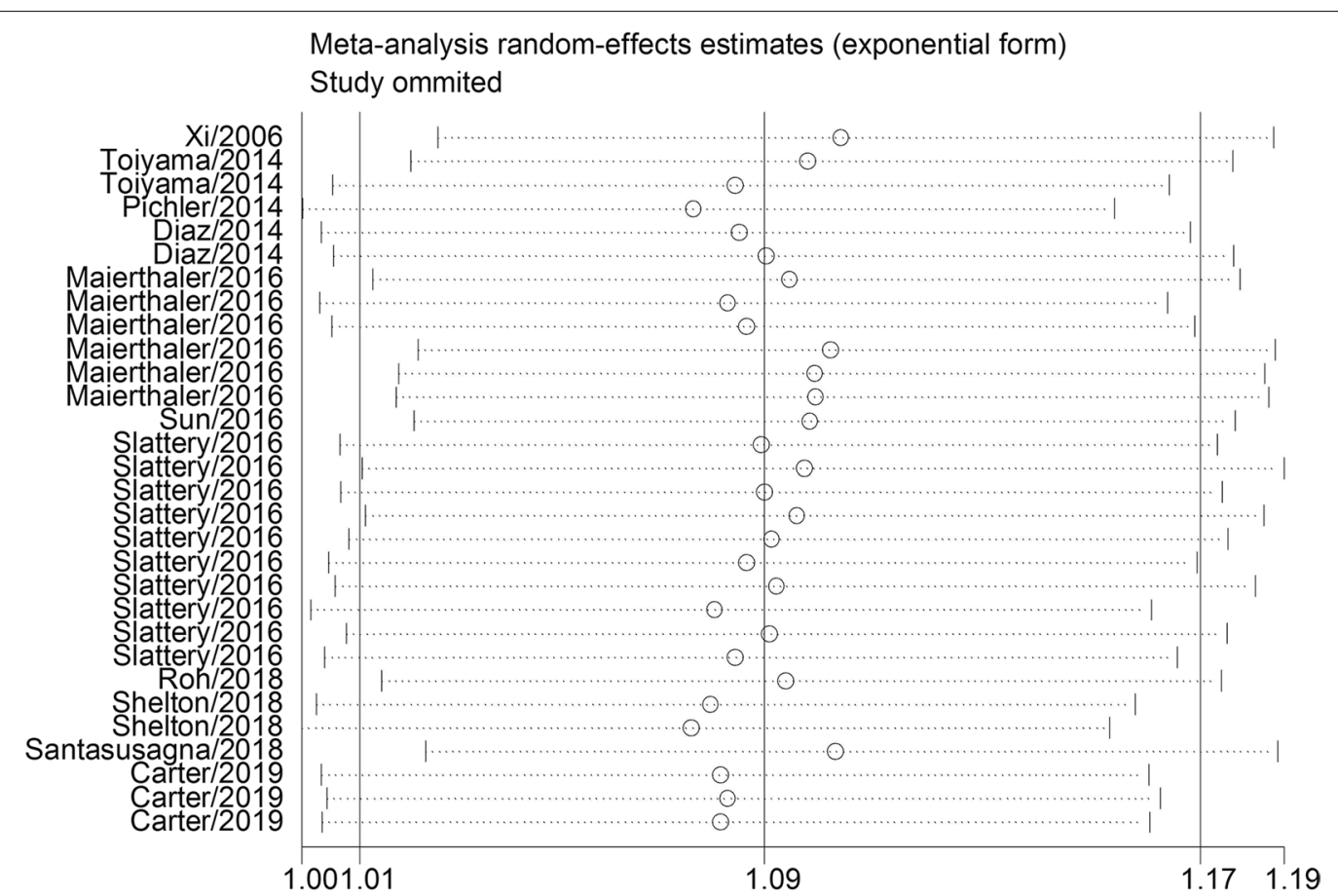

Fig. 3 Sensitivity analysis for the influence of individual studies on summarized hazard ratios

pathway, Proteoglycans in cancer, Focal adhesion and Viral carcinogenesis.

\section{Module screening and analysis}

In addition, the MCODE plug-in identified the most significant modules, and then conducted function and pathway enrichment analysis (Fig. 9). The most significant module for miR-200a network consisted of 12 nodes and 56 edges, which were primarily involved in miRNAs in cancer, pathways in cancer, colorectal cancer, FoxO signaling pathway, proteoglycans in cancer, cell cycle and Hippo signaling pathway. The most important module for miR-200b network contained 18 nodes and 73 edges, which were mainly linked with miRNAs in cancer, 


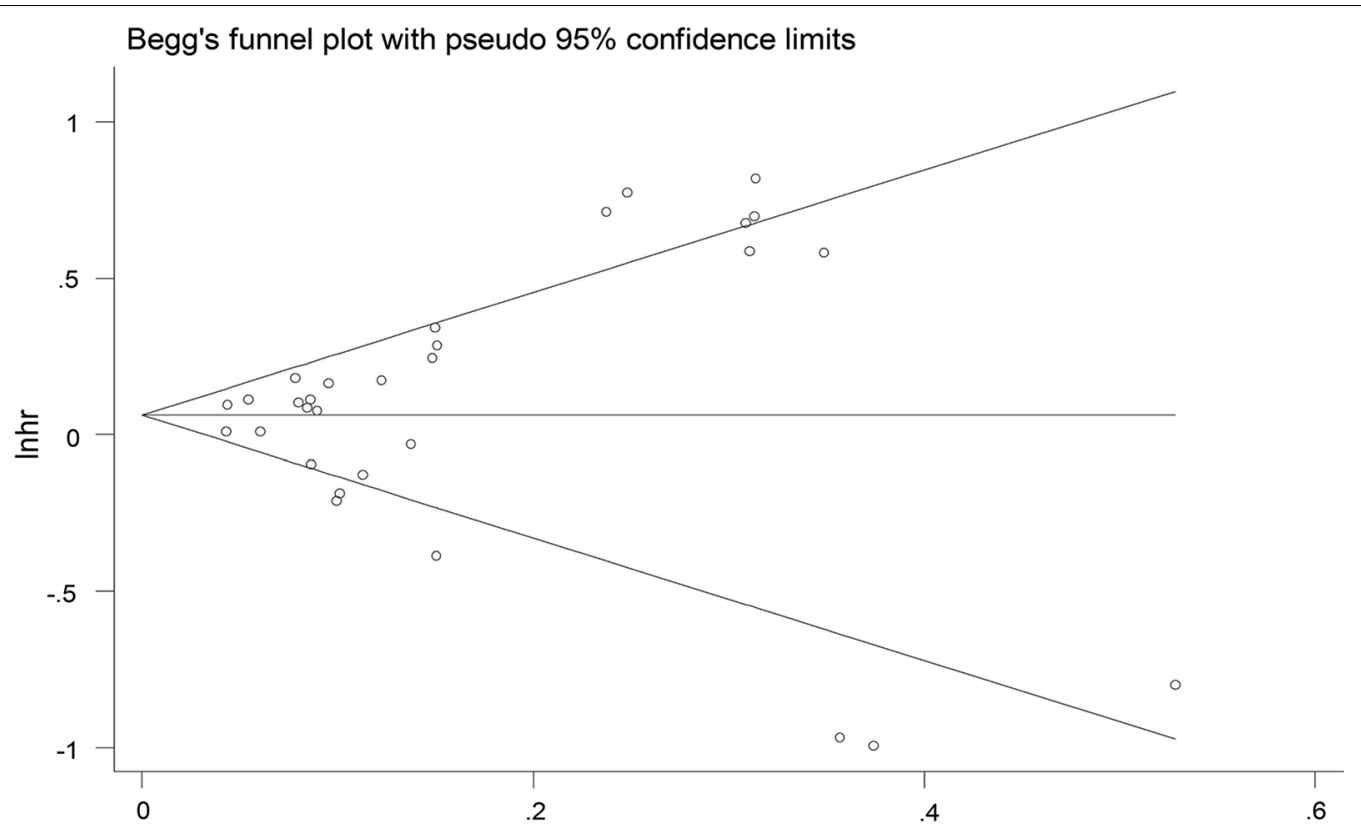

s.e. of: Inhr

Fig. 4 Funnel plot for publication bias analysis

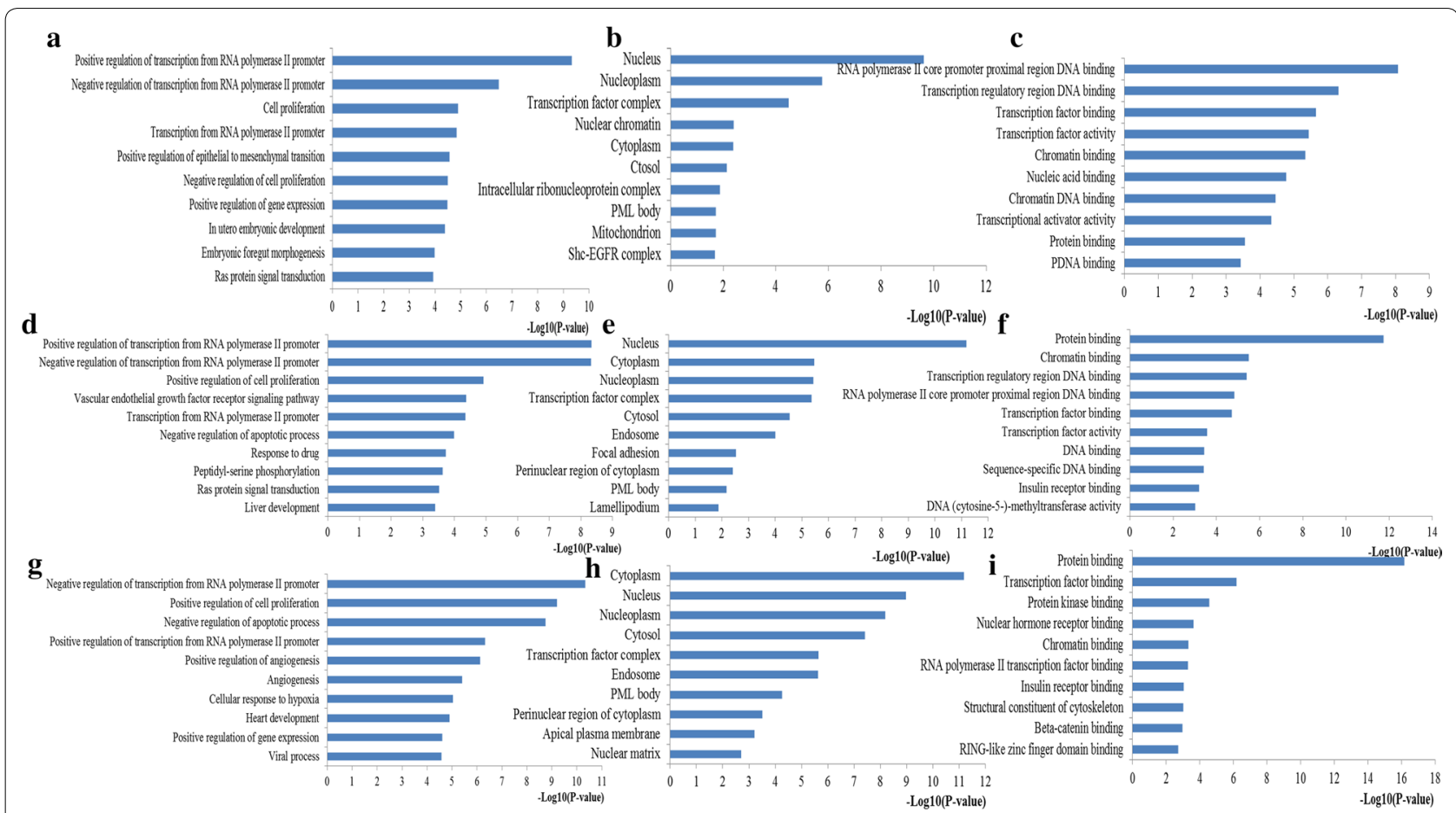

Fig. 5 Top ten GO annotation of miR-200s target genes. a Biological processes for miR-200a; b Cell component for miR-200a; c Molecular function for miR-200a; d Biological processes for miR-200b; e Cell component for miR-200b; f Molecular function for miR-200b; g Biological processes for miR-200c; $\boldsymbol{h}$ Cell component for miR-200c; i Molecular function for miR-200c 


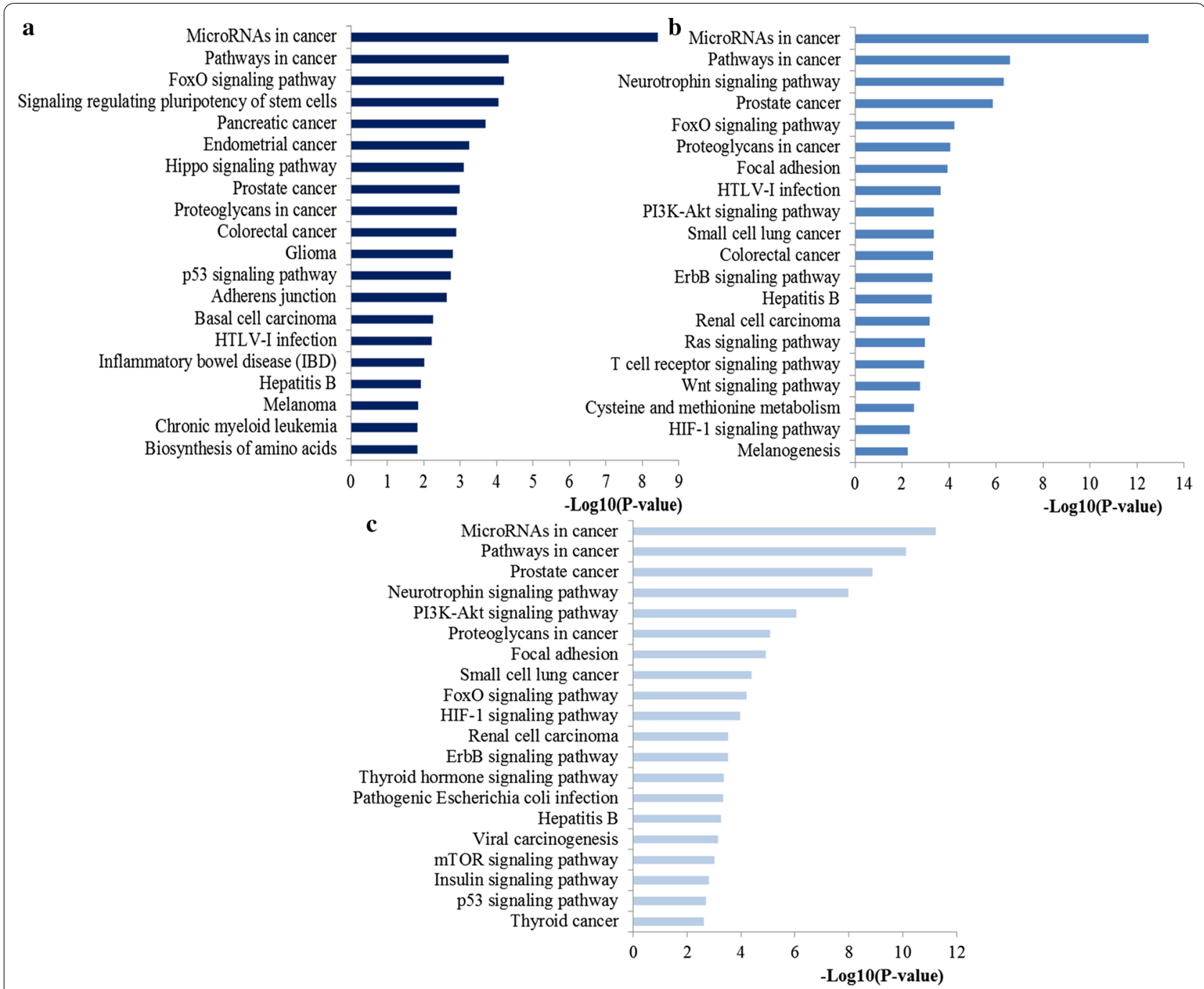

Fig. 6 Pathway enrichment results. a Top 20 pathways enriched by all the target genes of miR-200a; b Top 20 pathways enriched by the hub nodes of miR-200b; c Top 20 pathways enriched by the hub nodes of miR-200c

pathways in cancer, PI3K-Akt signaling pathway, focal adhesion, FoxO signaling pathway, and transcriptional misregulation in cancer. The most vital module for miR200c network including 15 nodes and 86 edges, which were highly associated with pathways in cancer, FoxO signaling pathway, miRNAs in cancer, PI3K-Akt signaling pathway, proteoglycans in cancer, Viral carcinogenesis, HIF-1 signaling pathway, cell cycle, and p53 signaling pathway.

\section{Discussion}

Numerous profiling studies have demonstrated that aberrant expression of miRNAs plays a significant role in the diagnosis and prognosis of human carcinomas including CRC (Additional file 1: Table S1). As one of the most representative miRNA members, miR-200s have been demonstrated by mounting evidence, indicating that miR-200s are noteworthy biomarkers for CRC prognosis. However, inconsistent results have emerged in different studies. Therefore, to clarify the prognostic value of miR200s as survival prediction biomarkers for CRC, data from published studies of miR-200s were systematically collected and assessed. We separately analyzed the biomarker roles of miR-200s through an integrated bioinformatics analysis to uncover their underlying mechanism involved in CRC.

We first quantitatively explored the prognostic role of miR-200s through a comprehensive meta-analysis. The result indicated that patients with a low miR-200s expression level were significantly correlated with shorter 


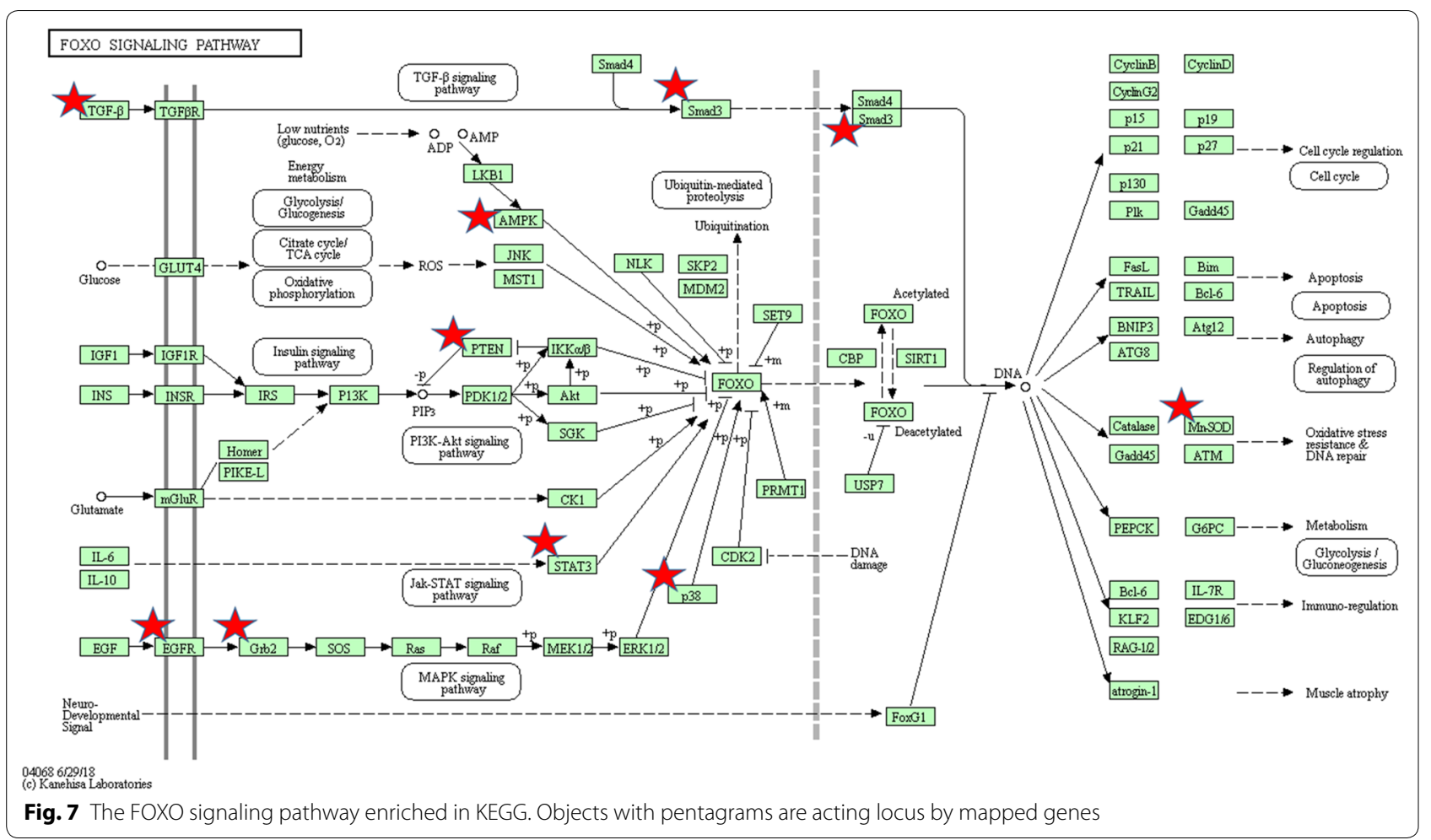

OS, compared than those with a high miR-200s expression level. Thus, it was preliminarily concluded that low miR-200s expression was closely related with worse OS in CRC. Afterwards, the subgroup analysis was selected to investigate the main factors that may contribute to the heterogeneity. The subgroup analysis results suggested that low expression of miR-200s was more significantly associated with worse OS of CRC patients among Americans than Asians and Europeans, which suggested that ethnicity may play an important part in association of miR-200s expression and CRC patient prognosis because of the variations in genetic background, environmental factors. According to the analysis results, it was also summarized that sample size had a significant impact on the predictive role of miR-200s which means that welldesigned studies with larger sample sizes are required to further improve the predictive role of miR-200s. Our results also suggested that the prognostic role of miR200 s varied for different types of sample types. Based on the analysis results, tissue samples could be considered as better sample source for classifying miR-200s as the molecular predictive biomarker of CRC. The results from subgroup studies by miR-200s classification revealed that the subgroup of miR-200a emerged statistically significantly higher predictive accuracy than miR-200b and miR-200c. Moreover, the meta-regression was also performed to seek the potential sources of heterogeneity; however, no significant findings were identified. In addition, sensitivity analysis demonstrated the robustness of our study. Promisingly, no obvious publication bias was found.

To further qualitatively explore the potential biological and molecular mechanisms associated with miR200s, bioinformatics analysis was applied to investigate the underlying functions, pathways, and networks of the genes. The GO analysis indicated that the target genes of miR-200a, miR-200b and miR-200c were mainly involved in regulating some important cellular processes at the biological process level, significantly enriched in some key cell structures for the cellular component level, and highly involved in binding function regarding the molecular function level, which primarily revealed the function of miR-200s involved in CRC initiation and progression. A KEGG analysis further explored the function of miR$200 \mathrm{~s}$ and revealed that the target genes of miR-200a, $200 \mathrm{~b}$ and miR-200c were particularly enriched in some important pathways. Most of identified pathways have been demonstrated to play important roles in the occurrence and development according to previous studies. For example, miRNAs in cancer, pathways in cancer, and colorectal cancer pathways intuitively reflected the relationships among miR-200s and CRC. Moreover, proteoglycans have been widely recognized as communication molecules which produce a marked effect in the invasion 

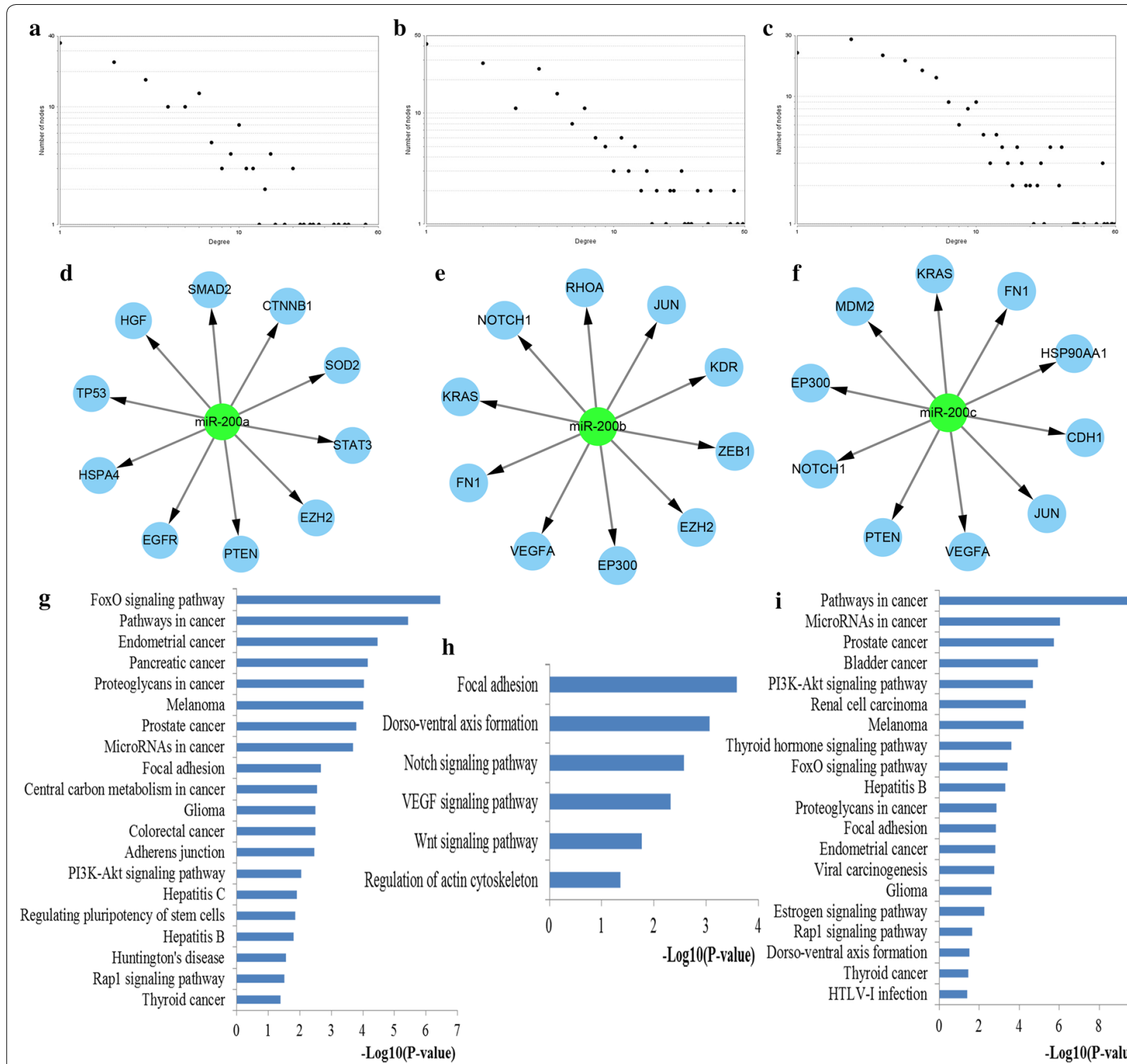

i Pathways in cancer MicroRNAs in cancer Prostate cancer Bladder cancer PI3K-Akt signaling pathway Renal cell carcinoma
Melanoma Thyroid hormone signaling pathway FoxO signaling pathway
Hepatitis $\mathrm{B}$

Proteoglycans in cancer Focal adhesion Endometrial cancer Viral carcinogenesis
Glioma

Estrogen signaling pathway Rapl signaling pathway Thyroid cancer
HTLV-I infection $\begin{array}{llllrr}0 & 2 & 4 & 6 & 8 & 10 \\ & & & \\ & & & \end{array}$

Fig. 8 PPI network construction results. a Degree distributions of nodes for the network set up with miR-200a targets; $\mathbf{b}$ Degree distributions of nodes for the network set up with miR-200b targets; c Degree distributions of nodes for the network set up with miR-200c targets; $\mathbf{d}$ Hub genes of the network for miR-200a targets; e Hub genes of the network for miR-200b targets; $\mathbf{f}$ Hub genes of the network for miR-200c targets; $\mathbf{g}$ Pathway enrichment results for the selected hub genes of miR-200a targets network; $\mathbf{h}$ Pathway enrichment results for the selected hub genes of miR-200b targets network; $\mathbf{i}$ Pathway enrichment results for the selected hub genes of miR-200c targets network

and growth and metastatic properties of cancerous cells [34]. Accumulating evidence indicates that proteoglycans have a significant impact on the behavior of cancer cells and the microenvironment during the progression of carcinogenesis and may serve as attractive pharmacological targets in cancer immunotherapy [35]. FoxO signaling plays a pivotal functional role as regulator of cellular homeostasis and putative tumor suppressor [36]. Targeting FoxO signaling may represent a potential approach to treat cancer [37]. Focal adhesion kinase, as a tyrosine kinase with high expressions in cancer cells, has been identified highly involved in the progression of tumors to a malignant phenotype [38]. Converging evidence have indicated that p53 signaling play an important role in various transcriptional and non-transcriptional biological processes such as cell proliferation, senescence, DNA repair, and cell death [39]. Previous studies have demonstrated that PI3K-Akt signaling serves 

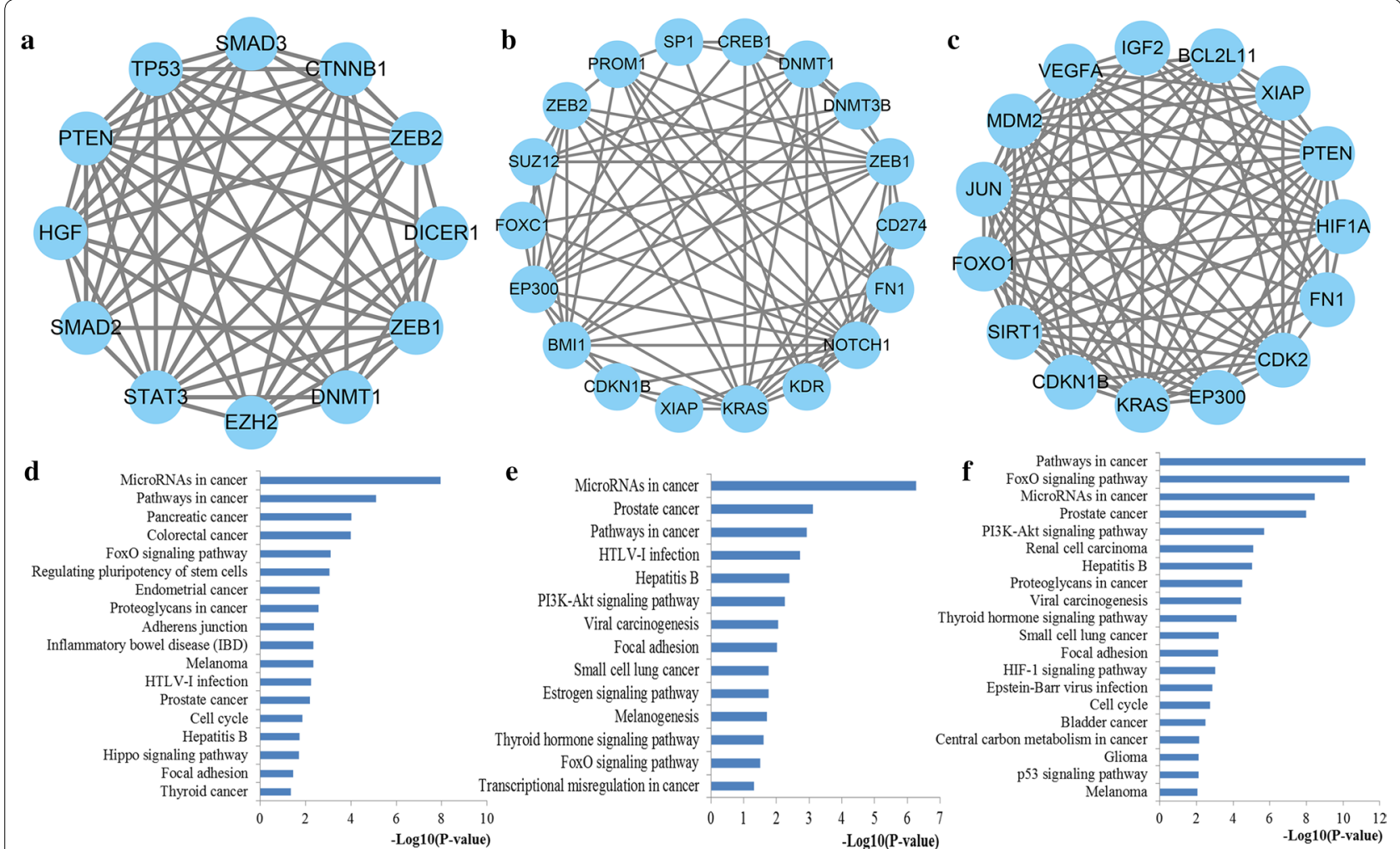

Fig. 9 Module analysis results of the PPI network. a The most significant module in the PPI network for miR-200a targets; $\mathbf{b}$ The most significant module in the PPI network for miR-200b targets; c The most significant module in the PPI network for miR-200c targets; $\mathbf{d}$ Pathways enriched by all the nodes involved in the identified module for miR-200a; e Pathways enriched by all the nodes involved in the identified module for miR-200b; $\mathbf{f}$ Pathways enriched by all the nodes involved in the identified module for miR-200c

as crucial coordinator of intracellular signaling including cell growth, differentiation, migration, and survival, as well as angiogenesis and metabolism [40]. Increasing evidence has confirmed the important part of Wnt signaling in a great diversity of cellular activities, including embryonic stem-cell development, tissue regeneration, cell differentiation, and immune cell regulation [41]. The RAS signaling regulates cancer cell proliferation, apoptosis, inflammation, migration, and metastasis [42]. The aberrant RAS signaling may cause carcinogenesis and targeting it may bring substantial improvement in clinical outcome in metastatic colorectal cancers [43]. It is well established that mTOR signaling, a critical protein in regulating cancer cells, takes part in varieties of fundamental biological activities containing cell death, autophagy, metabolic reprogramming, cell growth, and cell cycle [44]. HIF-1, a transcription factor with important part in response to low oxygen concentrations, or hypoxia, play significant roles in normal tissue development and function and cancer progression [45]. Activation of the HIF-1 pathway has been considered as an attractive therapeutic target [46]. The Hippo signaling, made up of a core kinases module and transcriptional activation module, has recently emerged as an important biochemical signaling in regulating a variety of signaling processes such as organ development and maintenance of tissue homeostasis [47]. The mechanism of miR-200s affecting the metastasis and unfavorable prognosis of CRC patients may be partly understood.

PPI network analysis was applied to investigate the most important target genes for miR-200s in CRC. The top network nodes with high degrees of betweenness centrality and closeness centrality and degree centrality were considered as the hub genes. To further validate the potential roles of the hub genes in CRC, KEGG pathway enrichment analysis was conducted. Results from KEGG analysis demonstrated that these hub genes were also significantly associated with some vital pathways. Most of the pathways have been proved by previous biological experiments. Aside from the above mentioned pathways, Notch signaling pathway has been identified as a highly conserved system in various multicellular organisms that is crucial for driving T-cell fate decisions, proliferation, and aberrant growth [48]. Moreover, VEGF signaling is a pivotal coordinator in the development of tumor angiogenesis, and inhibiting VEGF may provide a 
promising approach for tumor vessel normalization [49]. From the findings of the PPI analysis, it might be possible to hypothesize that miR-200s have a role in the initiation and progression of CRC by targeting these hub genes involved these key pathways, which may provide potential therapeutic targets.

The network module may determine the stability and function of the network. Correspondingly, the most significant modules of the networks for the targets of miR-200s were identified. KEGG pathway analysis revealed that the genes consisting of the networks for miR-200a, miR-200b and miR-200c were mainly associated with a series of well-studied pathways. In addition to the majority of these pathways that proved by literature exploration, another part have to mention is that the well-studied cell cycle pathway has been a vital pathway mediating every aspect of various fundamental cellular processes such as cell proliferation, apoptosis, differentiation, and migration [50]. Module analysis demonstrated that miR-200s were highly associated with the initiation and development of CRC again.

$\mathrm{CRC}$ is a heterogeneous disease and its initiation and progression may be affected by various confounding factors. It is deserved to be mentioned that miR-200a, miR-200b and miR-200c were enriched into the similar functional ontology, pathways, modules or networks and afterwards become more consistent, although they located at different chromosomal regions and regulated different types of genes. By convention, function associated miRNAs may exhibit a coordinated expression to play their role within the same functional modules, suggesting that miR-200a, miR-200b and miR-200c could have a synergistic effect to serve as an effective biomarker during the occurrence and development of CRC [51].

It should be noted that there were several limitations in our study. First, although we have conducted a comprehensive and systematic literature search and enrolled as many documents as possible, the size of available samples for evidence synthesis was still relatively small. More relevant studies and patients would be necessary to improve the reliability and confidence of our findings. Second, the cutoff definitions for high miR-200s expression were not precisely acknowledged among the studies, which could cause potential heterogeneity. Third, although we had planned to consider the impact of several important factors concerning heterogeneity such as age, sex and stage of cancer, this could not be accomplished due to the lack of access to the original data from the reviewed studies. Fourth, as drug resistance is a major problem with most of the cancer treatments. However, the enrolled studies in our study have not focused on the status of miR-200s in those CRC patients who developed resistant to therapy. Moreover, more and more findings have demonstrated that right-sided colon cancers and left-sided colon cancers are distinct clinical and biological entities and suggest that they should be treated as different diseases. However, most of the enrolled studies have not separated the status of miR-200s in tissue samples from different regions of colon in CRC patients. Lastly, our study certainly has its weaknesses due to the lack of experimental validation as such an experiment that focuses on the confirmation of the predictive power of our approach is difficult for us by now.

Nevertheless, we also need to stress some advantages in the present study. We combined two mainstream approaches including meta-analysis and bioinformatics analysis to answer whether miR-200s were associated with the prognosis of CRC patients and why miR-200s could be used as a prognostic biomarker for CRC. Several interesting results from our study may promote further investigation into the functions and partners of miR200s, push forward the clinical application of miR-200s and also provide us with more targets and strategies for therapy. Although not perfect, most of the findings from our results have been successfully confirmed by recent experimental literatures [34-51]. To better confirm the predictive power of our approach and understand the mechanisms underlying the initiation and progression of CRC, more large-scale and standard investigations are worth conducting.

\section{Conclusions}

In conclusion, based on meta-analysis and bioinformatics analysis results, our study demonstrated that miR200s could be promising biomarkers for CRC. However, further molecular and functional studies and large-scale controlled clinical studies are required to further explore the biomarker roles of miR-200s.

\section{Supplementary information}

Supplementary information accompanies this paper at https://doi. org/10.1186/s12935-020-1142-1.

Additional file 1: Table S1. miRNAs associated with CRC patient survival.

Abbreviations

CRC: Colorectal cancer; CT: Computed tomography; TNM: Tumor node metastasis; HR: Hazard ratio; Cl: Confidence interval; PRISMA: Preferred Reporting Items for Systematic Reviews and Meta-Analyses; NOS: Newcastle-Ottawa Scale; GO: Gene ontology; KEGG: Kyoto Encyclopedia of Genes and Genomes; PPI: Protein-protein interaction; DAVID: Database for annotation, visualization, and integrated discovery.

\section{Acknowledgements}

We would like to thank the authors of the primary studies.

\section{Authors' contributions}

QP and MC performed the retrieval of data. TL and XC took part in the statistical analysis for meta-analysis, YS and YZ were responsible for the 
bioinformatics analysis. BX drafted and revised the manuscript. All authors read and approved the final manuscript.

\section{Funding}

This work was supported by Jiangsu Commission of Health medical research project (H2018115), Suzhou Science and Technology Development Project (SYS2019059), Suzhou Introduction Project of Clinical Medical Expert Group (SZYJTD201804), Jiangsu Medical Innovation Team (CXDT-37) and Suzhou Clinical Medical Center Construction Project (Szzxj201503).

\section{Availability of data and materials}

The data supporting the conclusions of this article is within the article.

\section{Ethics approval and consent to participate}

Not applicable.

\section{Consent for publication}

All authors approve the publication of this manuscript.

\section{Competing interests}

The authors declare they have no competing interest.

\section{Author details \\ ${ }^{1}$ Department of Radiotherapy \& Oncology, The Second Affiliated Hospital of Soochow University, Suzhou, China. ${ }^{2}$ Institute of Radiotherapy \& Oncology, Soochow University, Suzhou, China. ${ }^{3}$ Department of General Surgery, The Second Affiliated Hospital of Soochow University, San Xiang Road No. 1055, Suzhou, Jiangsu 215004, China. ${ }^{4}$ Department of Radiation Oncology, The Affiliated Suzhou Science \& Technology Town Hospital of Nanjing Medical University, Suzhou, China.}

Received: 22 November 2019 Accepted: 12 February 2020

Published online: 18 February 2020

\section{References}

1. Siegel RL, Miller KD, Jemal A. Cancer statistics, 2019. CA Cancer J Clin. 2019;69(1):7-34.

2. Miller KD, Nogueira L, Mariotto AB, et al. Cancer treatment and survivorship statistics, 2019. CA Cancer J Clin. 2019;69(5):363-85.

3. Dekker E, Tanis PJ, Vleugels JLA, Kasi PM, Wallace MB. Colorectal cancer. Lancet. 2019;394(10207):1467-80.

4. Sveen A, Kopetz S, Lothe RA. Biomarker-guided therapy for colorectal cancer: strength in complexity. Nat Rev Clin Oncol. 2019;17(1):11-32.

5. Yates LA, Norbury CJ, Gilbert RJ. The long and short of microRNA. Cell. 2013;153(3):516-9.

6. Lin S, Gregory RI. MicroRNA biogenesis pathways in cancer. Nat Rev Cancer. 2015;15(6):321-33.

7. Sun Z, Shi K, Yang S, et al. Effect of exosomal miRNA on cancer biology and clinical applications. Mol Cancer. 2018;17(1):147.

8. Liu W, Zhang K, Wei P, et al. Correlation between miR-200 family overexpression and cancer prognosis. Dis Markers. 2018;2018:6071826.

9. Yu C, Wan H, Shan R, et al. The prognostic value of the MiR-200 family in colorectal cancer: a meta-analysis with 1882 patients. J Cancer. 2019;10(17):4009-16.

10. Page MJ, Moher D. Evaluations of the uptake and impact of the preferred reporting items for systematic reviews and meta-analyses (PRISMA) statement and extensions: a scoping review. Syst Rev. 2017;6(1):263.

11. Wang Y, Zeng T. Response to: practical methods for incorporating summary time-to-event data into meta-analysis. Trials. 2013;14:391.

12. Stang A. Critical evaluation of the Newcastle-Ottawa scale for the assessment of the quality of nonrandomized studies in meta-analyses. Eur J Epidemiol. 2010;25(9):603-5.

13. Higgins JP, Thompson SG, Deeks JJ, Altman DG. Measuring inconsistency in meta-analyses. BMJ. 2003;327(7414):557-60.

14. Mueller M, D'Addario M, Egger $M$, et al. Methods to systematically review and meta-analyse observational studies: a systematic scoping review of recommendations. BMC Med Res Methodol. 2018;18(1):44.
15. Augusteijn HEM, van Aert RCM, van Assen M. The effect of publication bias on the $Q$ test and assessment of heterogeneity. Psychol Methods. 2019;24(1):116-34

16. Hsu SD, Lin FM, Wu WY, et al. miRTarBase: a database curates experimentally validated microRNA-target interactions. Nucleic Acids Res. 2011;39(Database issue):D163-9.

17. Huang HY, Lin YC, Li J, et al. miRTarBase 2020: updates to the experimentally validated microRNA-target interaction database. Nucleic Acids Res. 2019:48(D1):D148-54.

18. The Gene Ontology C. The Gene Ontology Resource: 20 years and still GOing strong. Nucleic Acids Res. 2019;47(D1):D330-8.

19. Kanehisa M, Sato Y, Furumichi M, Morishima K, Tanabe M. New approach for understanding genome variations in KEGG. Nucleic Acids Res. 2019;47(D1):D590-5.

20. Dennis G Jr, Sherman BT, Hosack DA, et al. DAVID: database for annotation, visualization, and integrated discovery. Genome Biol. 2003:4(5):P3.

21. Szklarczyk D, Gable AL, Lyon D, et al. STRING v11: protein-protein association networks with increased coverage, supporting functional discovery in genome-wide experimental datasets. Nucleic Acids Res. 2019:47(D1):D607-13.

22. Tang Y, Li M, Wang J, Pan Y, Wu FX. CytoNCA: a cytoscape plugin for centrality analysis and evaluation of protein interaction networks. Bio Syst. 2015;127:67-72.

23. Diaz T, Tejero R, Moreno I, et al. Role of miR-200 family members in survival of colorectal cancer patients treated with fluoropyrimidines. J Surg Oncol. 2014;109(7):676-83.

24. Pichler M, Ress AL, Winter E, et al. MiR-200a regulates epithelial to mesenchymal transition-related gene expression and determines prognosis in colorectal cancer patients. Br J Cancer. 2014;110(6):1614-21.

25. Slattery ML, Herrick JS, Mullany LE, et al. An evaluation and replication of miRNAs with disease stage and colorectal cancer-specific mortality. Int J Cancer. 2015;137(2):428-38.

26. Toiyama Y, Hur K, Tanaka K, et al. Serum miR-200c is a novel prognostic and metastasis-predictive biomarker in patients with colorectal cancer. Ann Surg. 2014;259(4):735-43.

27. Sun Y, Liu Y, Cogdell D, et al. Examining plasma microRNA markers for colorectal cancer at different stages. Oncotarget. 2016;7(10):11434-49.

28. Maierthaler M, Benner A, Hoffmeister M, et al. Plasma miR-122 and miR-200 family are prognostic markers in colorectal cancer. Int J Cancer. 2017;140(1):176-87.

29. Roh MS, Lee HW, Jung SB, et al. Expression of miR-200c and its clinicopathological significance in patients with colorectal cancer. Pathol Res Pract. 2018;214(3):350-5.

30. Santasusagna S, Moreno I, Navarro A, et al. Prognostic impact of miR-200 family members in plasma and exosomes from tumor-draining versus peripheral veins of colon cancer patients. Oncology. 2018;95(5):309-18.

31. Shelton PM, Duran A, Nakanishi Y, et al. The Secretion of miR-200s by a PKCzeta/ADAR2 signaling axis promotes liver metastasis in colorectal cancer. Cell Rep. 2018;23(4):1178-91.

32. Carter JV, O'Brien SJ, Burton JF, et al. The microRNA-200 family acts as an oncogene in colorectal cancer by inhibiting the tumor suppressor RASSF2. Oncol Lett. 2019;18(4):3994-4007.

33. Xi Y, Formentini $A$, Chien $M$, et al. Prognostic values of microRNAs in colorectal cancer. Biomark Insights. 2006;2:113-21.

34. $\mathrm{Xu} L$, Tang $\mathrm{L}$, Zhang L. Proteoglycans as miscommunication biomarkers for cancer diagnosis. Prog Mol Biol Transl Sci. 2019;162:59-92.

35. Espinoza-Sanchez NA, Gotte M. Role of cell surface proteoglycans in cancer immunotherapy. Semin Cancer Biol. 2019. https://doi.org/10.1016/j. semcancer.2019.07.012.

36. Ma J, Matkar S, He X, Hua X. FOXO family in regulating cancer and metabolism. Semin Cancer Biol. 2018;50:32-41.

37. Farhan $\mathrm{M}$, Wang H, Gaur U, Little PJ, X U J, Zheng W. FOXO signaling pathways as therapeutic targets in cancer. Int J Biol Sci. 2017;13(7):815-27.

38. Zhou J, Yi Q, Tang $L$. The roles of nuclear focal adhesion kinase (FAK) on cancer: a focused review. J Exp Clin Cancer Res. 2019;38(1):250.

39. Bykov VJN, Eriksson SE, Bianchi J, Wiman KG. Targeting mutant p53 for efficient cancer therapy. Nat Rev Cancer. 2018;18(2):89-102.

40. Hoxhaj G, Manning BD. The PI3K-AKT network at the interface of oncogenic signalling and cancer metabolism. Nat Rev Cancer. 2019;20(2):74-88 
41. Haseeb M, Pirzada RH, Ain QU, Choi S. Wnt signaling in the regulation of immune cell and cancer therapeutics. Cells. 2019;8(11):1380.

42. Soleimani A, Rahmani F, Saeedi N, et al. The potential role of regulatory microRNAs of RAS/MAPK signaling pathway in the pathogenesis of colorectal cancer. J Cell Biochem. 2019;120(12):19245-53.

43. Ciliberto D, Staropoli N, Caglioti F, et al. The best strategy for RAS wildtype metastatic colorectal cancer patients in first-line treatment: a classic and bayesian meta-analysis. Crit Rev Oncol Hematol. 2018;125:69-77.

44. Murugan AK. mTOR: Role in cancer, metastasis and drug resistance. Semin Cancer Biol. 2019;59:92-111.

45. Pezzuto A, Carico E. Role of HIF-1 in cancer progression: novel insights. A review. Curr Mol Med. 2018;18(6):343-51.

46. Karagiota A, Kourti M, Simos G, Mylonis I. HIF-1alpha-derived cellpenetrating peptides inhibit ERK-dependent activation of HIF-1 and trigger apoptosis of cancer cells under hypoxia. Cell Mol Life Sci. 2019;76(4):809-25.

47. Yeung YT, Guerrero-Castilla A, Cano M, Munoz MF, Ayala A, Arguelles S. Dysregulation of the Hippo pathway signaling in aging and cancer. Pharmacol Res. 2019;143:151-65.
48. Bigas A, Espinosa L. The multiple usages of Notch signaling in development, cell differentiation and cancer. Curr Opin Cell Biol. 2018;55:1-7.

49. Mercurio AM. VEGF/neuropilin signaling in cancer stem cells. Int J Mol Sci. 2019;20(3):490.

50. Icard P, Fournel L, Wu Z, Alifano M, Lincet $H$. Interconnection between metabolism and cell cycle in cancer. Trends Biochem Sci. 2019;44(6):490-501.

51. Peng Q, Shen Y, Lin K, Zou L, Shen Y, Zhu Y. Comprehensive and integrative analysis identifies microRNA-106 as a novel non-invasive biomarker for detection of gastric cancer. J Transl Med. 2018;16(1):127.

\section{Publisher's Note}

Springer Nature remains neutral with regard to jurisdictional claims in published maps and institutional affiliations.
Ready to submit your research? Choose BMC and benefit from:

- fast, convenient online submission

- thorough peer review by experienced researchers in your field

- rapid publication on acceptance

- support for research data, including large and complex data types

- gold Open Access which fosters wider collaboration and increased citations

- maximum visibility for your research: over $100 \mathrm{M}$ website views per year

At BMC, research is always in progress.

Learn more biomedcentral.com/submissions 